NASA/CR-2000-210226

AIAA-2000-2059

\title{
Influence of Mean-Density Gradient on Small-Scale Turbulence Noise
}

Abbas Khavaran

Dynacs Engineering Company, Inc., Brook Park, Ohio 
The NASA STI Program Office ... in Profile

Since its founding, NASA has been dedicated to the advancement of aeronautics and space science. The NASA Scientific and Technical Information (STI) Program Office plays a key part in helping NASA maintain this important role.

The NASA STI Program Office is operated by Langley Research Center, the Lead Center for NASA's scientific and technical information. The NASA STI Program Office provides access to the NASA STI Database, the largest collection of aeronautical and space science STI in the world. The Program Office is also NASA's institutional mechanism for disseminating the results of its research and development activities. These results are published by NASA in the NASA STI Report Series, which includes the following report types:

- TECHNICAL PUBLICATION. Reports of completed research or a major significant phase of research that present the results of NASA programs and include extensive data or theoretical analysis. Includes compilations of significant scientific and technical data and information deemed to be of continuing reference value. NASA's counterpart of peerreviewed formal professional papers but has less stringent limitations on manuscript length and extent of graphic presentations.

- TECHNICAL MEMORANDUM. Scientific and technical findings that are preliminary or of specialized interest, e.g., quick release reports, working papers, and bibliographies that contain minimal annotation. Does not contain extensive analysis.

- CONTRACTOR REPORT. Scientific and technical findings by NASA-sponsored contractors and grantees.
- CONFERENCE PUBLICATION. Collected papers from scientific and technical conferences, symposia, seminars, or other meetings sponsored or cosponsored by NASA.

- SPECIAL PUBLICATION. Scientific, technical, or historical information from NASA programs, projects, and missions, often concerned with subjects having substantial public interest.

- TECHNICAL TRANSLATION. Englishlanguage translations of foreign scientific and technical material pertinent to NASA's mission.

Specialized services that complement the STI Program Office's diverse offerings include creating custom thesauri, building customized data bases, organizing and publishing research results ... even providing videos.

For more information about the NASA STI Program Office, see the following:

- Access the NASA STI Program Home Page at http://www.sti.nasa.gov

- E-mail your question via the Internet to help@sti.nasa.gov

- Fax your question to the NASA Access Help Desk at (301) 621-0134

- Telephone the NASA Access Help Desk at (301) 621-0390

- Write to: NASA Access Help Desk NASA Center for AeroSpace Information 7121 Standard Drive Hanover, MD 21076 
NASA/CR-2000-210226

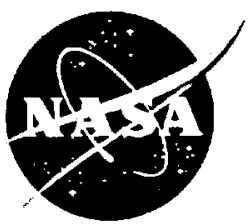

\section{Influence of Mean-Density Gradient on Small-Scale Turbulence Noise}

Abbas Khavaran

Dynacs Engineering Company, Inc., Brook Park, Ohio

Prepared for the

6th Aerocoustics Conference and Exhibit

cosponsored by the American Institute of Aeronautics and Astronautics

and the Confederation of European Aerospace Societies

Lahaina, Hawaii, June 12-14, 2000

Prepared under Contract NAS3-98008

National Aeronautics and

Space Administration

Glenn Research Center 


\section{Acknowledgments}

This work was sponsored by NASA Glenn Research Center under the contract NAS3-98008.

Available from

NASA Center for Aerospace Information 7121 Standard Drive Hanover, MD 21076

Price Code: A03
National Technical Information Service 5285 Port Royal Road Springfield, VA 22100 


\title{
Influence of Mean-Density Gradient on Small-Scale Turbulence Noise
}

\author{
Abbas Khavaran \\ Dynacs Engineering, Inc. \\ NASA Glenn Research Center \\ Cleveland, OH 44135-3191
}

\begin{abstract}
A physics-based methodology is described to predict jetmixing noise due to small-scale turbulence. Both self- and shear-noise source terms of Lilley's equation are modeled and the far-field aerodynamic noise is expressed as an integral over the jet volume of the source multiplied by an appropriate Green's function which accounts for source convection and mean-flow refraction. Our primary interest here is to include transverse gradients of the mean density in the source modeling. It is shown that, in addition to the usual quadrupole type sources which scale to the fourthpower of the acoustic wave number, additional dipole and monopole sources are present that scale to lower powers of wave number. Various two-point correlations are modeled and an approximate solution to noise spectra due to multipole sources of various orders is developed. Mean flow and turbulence information is provided through RANS-k $\varepsilon$ solution. Numerical results are presented for a subsonic jet at a range of temperatures and Mach numbers. Predictions indicated a decrease in high frequency noise with added heat, while changes in the low frequency noise depend on jet velocity and observer angle.
\end{abstract}

\section{Introduction}

Development of a reliable and robust jet noise prediction method is instrumental in the design of jet noise abatement tools. Short of Computational Aeroacoustics (CAA) which is arguably the hope of the future $[1,2]$, most near-term approaches to the jet noise problem start with some derivative of Lighthill's theory in order to describe the sources of aerodynamic noise as well as the propagation effects (e.g. chapter 6, Ref. 3). Pilon and Morris [4] recently developed a semi-analytical method for jet noise prediction that employs scaling laws based on acoustic analogy to characterize the quadrupole and dipole sources and uses a simplified ray-acoustic approximation to propagate the sound to the far field. Tam et al. [5] concluded that a two-component spectra is needed to describe the noise generation due to both small-scale turbulence and instability waves in the jet flow. They maintain that instability waves generate sound at subsonic as well as supersonic speeds, although they become increasingly more efficient at high supersonic speeds.

Copyright $(2000$ by the American Instirute of Aeronautics and Astronautics, Inc. No copyright is asserted in the United States under Title 17. U.S. Code. The U.S. Government has a royalty-free license to exercise all rights under the copyright claimed herein for Governmental Purposes. All other rights are reserved by the copyright owner.
More recently, Tam and Auriault [6] developed a semi-empirical theory to predict the small-scale turbulence noise based on the kinetic theory of gases. They argue that the source of small-scale turbulence noise is the kinetic energy of fine-scale turbulence and use a Reynolds Averaged Navier-Stokes (RANS) solution with a $k \varepsilon$ turbulence model to obtain the source strength as well as the time- and length scales of small eddies.

Although, in principle, direct numerical simulation (DNS) may be used to describe the acoustic near field [7,8], all scales of motion cannot be resolved for the high Reynolds-number jets of practical interest due to increasing demand on grid resolution. It is argued that at supersonic speeds, large-scale structures dominate the initial noise-producing region of the jet. Consequently, large eddy simulation (LES) has been advocated $[9,10]$ as a useful and practical tool to predict the acoustically efficient larger scales while modeling the smaller subgridscale turbulence.

In the present study, we employ a physics-based modeling approach to describe the sound sources of smallscale turbulence. Our primary concems are the so-called self- and shear-noise sources of Lilley's equations, commonly represented as $\rho D \partial^{2}\left(u_{i} u_{j}\right) / \partial x_{i} \partial x_{j}$ and $-2 \rho(d U / d r) \partial^{2}\left(u_{2} u_{j}\right) / \partial x_{1} \partial x_{j}$ respectively. A great deal of simplification in modeling of a two-point correlation may be achieved by assuming that the mean density $\rho$ is constant (i.e. quasi-incompressible turbulence), and by considering turbulence as isotropic. Reference [11] details the derivation of various quadrupole type sources that emerge from permutations of a two-point velocity correlation using both isotropic and axisymmetric turbulence models. Here, we assume that the mean flow is locally parallel and its density is a function of the spanwise coordinate $r$. The radial gradient of the mean density (and sound speed) produces additional sources within both the self- and shear-noise terms. Approximate expressions for noise spectra from various source types is given and the significance of newly derived sources and their contribution to noise of heated jets is examined through a numerical simulation of a simple round jet.

On the experimental side, the effect of jet density on aerodynamic mixing noise from subsonic and fully expanded supersonic jets has been studied by a number of 
investigators. Hoch et al. [12] studied the effect of density on noise by varying the jet temperature at a range of velocities from 150 to $800 \mathrm{~m} / \mathrm{s}$. Their far-field measurements show the expected reduction in noise with reducing density at high velocities and the existence of a low velocity regime in which the reduction of density by heating the jet increases the acoustic power. A more detailed experimental study of the effect of jet density on noise spectra is given by Tanna [13]. According to his findings, at high Strouhal numbers, mixing noise decreases with heating the jet throughout the velocity range. At low source Strouhal numbers, the intensity increases with heating at subsonic acoustic Mach numbers $\left(U / a_{\infty}\right)$ while it decreases with heating at supersonic Mach numbers. We intend to compare our predictions with these observations using jets numerically calculated at inflow boundary conditions that could closely simulate the experiment.

For the most part, our predictions agree with data and indicate a reduction in high frequency noise with increasing jet temperature. At intermediate and low subsonic speeds, heating enhances the low frequency noise at smaller angle from jet inlet. In addition, predictions indicate that density gradients of the mean flow contribute primarily in the context of the dipole sources of self noise and radiate mainly at $90^{\circ}$.

The paper begins with the formulation of the goveming equations. In sec 2.1 we briefly describe Balsa's Green's function. Modeling of two-point correlations and sound spectral intensity due to various terms of self and shear noise are described in section 2.2. Sections 3 and 4 describe implementation of the prediction methodology and the final summary.

\section{Spectral Density of Sound as Related to Source and Green's Function}

We assume that the mean flow is locally parallel and that local density and sound speed are functions of the radial coordinate $r$ only

$$
U=U(r), \quad \rho=\rho(r), \quad a=a(r) .
$$

Lilley's equation for an inviscid flow linearized about a unidirectional transversely sheared mean flow is

$$
\begin{gathered}
L\left(p ; U, x_{1}\right) \equiv \frac{1}{a^{2}} D^{3} p-D \nabla^{2} p-\frac{d}{d r}\left(\log a^{2}\right) D \frac{\partial p}{\partial r} \\
+2 \frac{d U}{d r} \frac{\partial^{2} p}{\partial x_{1} \partial r} \\
=\underbrace{\rho D \frac{\partial^{2}\left(u_{i} u_{j}\right)}{\partial x_{i} \partial x_{j}}}_{\text {Self }- \text { Noise }}-\underbrace{2 \rho \frac{d U}{d r} \frac{\partial^{2}\left(u_{2} u_{j}\right)}{\partial x_{1} \partial x_{j}}}_{\text {Shear-Noise }}+\cdots,
\end{gathered}
$$

where $p$ is the acoustic pressure, $r, \varphi, x_{1}$ denote the cylindrical coordinates, $D=\partial / \partial t+U \partial / \partial x_{1}$ is the convective derivative, $\nabla^{2}$ is the Laplacian operator in a cylindrical coordinate system and $u_{i}$ is the fluctuating part of the velocity. The terms on the right-hand side of Eq. (2) are usually referred to as self- and shear-noise source terms, respectively.

\subsection{Moving Reference Frame (Balsa's Solution)}

To accoumt for both source convection and the refraction of sound in the presence of the mean flow, a closed form solution to Lilley's equation in the high frequency limit was discussed by Balsa in References 14, 15 and 16. Balsa's solution is expressed in a coordinate system $\vec{x}^{\prime}$ moving with the turbulent eddies.

$$
\begin{aligned}
& L\left(G e^{-i \Omega} ; V, x_{1}^{\prime}\right)=e^{-i \Omega t} \delta\left(x_{1}^{\prime}\right) \delta\left(\varphi-\varphi_{o}\right) \delta\left(r-r_{o}\right) / r, \\
& \vec{x}^{\prime}=\bar{x}-\vec{U}_{c} t, V=U-U_{c}, D=\partial / \partial t+V \partial / \partial x_{1}^{\prime} .
\end{aligned}
$$

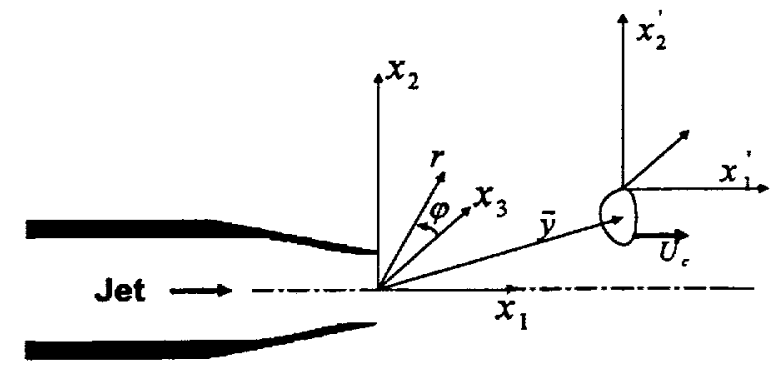

Fig. 1 Moving frame

The convection velocity $\bar{U}_{c}$ is in the $x_{1}$ direction and is usually some fraction of jet exit velocity. Here, $\Omega$ refers to the source frequency in a moving reference frame, i.e., a frame moving with a convecting eddy. A two-point space-time correlation is denoted as $R(\vec{y}, \vec{\xi}, \tau)$ where $\bar{\xi}$ and $\tau$ represent the spatial and temporal separation between points $\vec{y}_{1}=\vec{y}-\vec{\xi} / 2$ and $\vec{y}_{2}=\vec{y}+\vec{\xi} / 2$. Experimentally, the correlation $R(\vec{y}, \vec{\xi}, \tau)$ in a jet flow describes a fluctuating pattern in a moving frame and is expressed as

$$
R(\vec{y}, \bar{\xi}, \tau)=R_{m}\left(\vec{y}, \vec{\xi}_{m}, \tau\right), \quad \vec{\xi}_{m}=\vec{\xi}-U_{c} \tau
$$

Far-field spectral density becomes

$$
\begin{aligned}
\overline{p^{2}}\left(\vec{x}^{\prime}, \Omega\right) & =\int_{\vec{y}} \int_{\xi_{m}} G^{*}\left(\vec{x}^{\prime}, \vec{y}-\vec{\xi} / 2, \Omega\right) G\left(\vec{x}^{\prime}, \vec{y}+\vec{\xi} / 2, \Omega\right) \\
& \times Q_{m}\left(\vec{y}, \vec{\xi}_{m}, \Omega\right) d \bar{\xi}_{m} \not \vec{y}
\end{aligned}
$$

where * indicates a complex conjugate and $Q_{m}\left(\vec{y}, \vec{\xi}_{m}, \Omega\right)$ is the spectral density of a two-point space-time correlation in a moving frame 


$$
Q_{m}\left(\vec{y}, \bar{\xi}_{m}, \Omega\right)=\int_{-\infty}^{+\infty} R_{m}\left(\vec{y}, \bar{\xi}_{m}, \tau\right) e^{i \Omega \tau} d \tau .
$$

A Doppler factor relates source frequency $\Omega$ to the observer frequency $\omega$.

For brevity, we shall suppress subscript $m$ in writing source correlation functions and use $\bar{\xi}$ as a separation vector with respect to a moving frame in the remaining discussions. It turns out that the Green's function solution to Eq. (3) depends on the behavior of a so-called shielding function $g$ in the vicinity of the source. The shielding function is defined as

$$
g^{2}(r, \theta)=\frac{(1-M \cos \theta)^{2}\left(a_{\infty} / a\right)^{2}-\cos ^{2} \theta}{\left(1-M_{c} \cos \theta\right)^{2}},
$$

where $M=U(r) / a_{\infty}, M_{c}=U_{c} / a_{\infty}$ is the source convection Mach number, $\theta$ is the polar angle from downstream jet axis and subscript $\infty$ refers to ambient. The zeros of $g^{2}$, denoted as $r_{\sigma}$, are called the turning points. Shielding of radiated sound depends on the number of turning points in the vicinity of a source. When there are no turning points (i.e. $g^{2}>0$ ); the Green's function solution to Eq. (3) (referred to as an unshielded solution) is given $^{14,15,16}$ as

$$
\begin{aligned}
G\left(\vec{x}^{\prime}, \bar{y}, \Omega\right)= & \frac{i}{4 \pi a_{\infty} k R} \frac{a_{o} / a_{\infty}}{\left(1-M_{o} \cos \theta\right)^{2}}\left(\frac{\zeta_{o}}{r_{o} g_{o}}\right)^{1 / 2} e^{i \Omega R / a_{\infty}} \\
& \times e^{i k\left[\int_{0}^{\infty}\left(g-g_{\infty}\right) d r-\zeta_{o} \cos \left(\varphi-\varphi_{0}\right)\right]},
\end{aligned}
$$

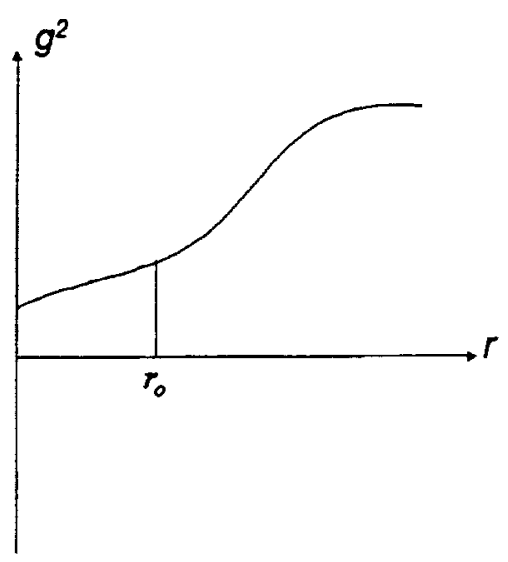

Fig. 2 No turning point problem.

and

$$
\begin{aligned}
& \zeta=\int_{0}^{r} g(r, \theta) d r, \quad k=\Omega / \mathrm{a}_{\infty}, \\
& g_{\infty} \equiv g(r \rightarrow \infty, \theta)=\sin \theta /\left(1-M_{c} \cos \theta\right) .
\end{aligned}
$$

The above Green's finction was obtained for a far-field observer (i.e. $R / D>>1$ ). When the shielding function crosses zero only once, i.e. $g\left(r_{\sigma}, \theta\right)=0$, and if the source is located at $r_{0}<r_{\sigma}$, the shielding function $g^{2}(r, \theta)$ becomes negative for $r<r_{\sigma}$. The Green's function is then expressed as ${ }^{14,15}$

$$
\begin{aligned}
G\left(\vec{x}^{\prime}, \vec{y}, \Omega\right)= & \frac{i}{4 \pi a_{\infty} k R} \frac{a_{o} / a_{\infty}}{\left(1-M_{o} \cos \theta\right)^{2}}\left(\frac{\zeta_{0}}{r_{o} f_{o}}\right)^{1 / 2} e^{i \Omega R / a_{\infty}} \\
& \times e^{i k\left[\int_{r_{0}}^{\infty}\left(g-g_{\infty}\right) d r-\delta_{\infty} r_{o}\right]} e^{-k\left[\zeta_{o}-\zeta_{0} \cos \left(\phi-\varphi_{0}\right)\right]},(7 c)
\end{aligned}
$$

where

$$
f^{2}=-g^{2}, \quad \zeta=\int_{0}^{r} f(r, \theta) d r .
$$

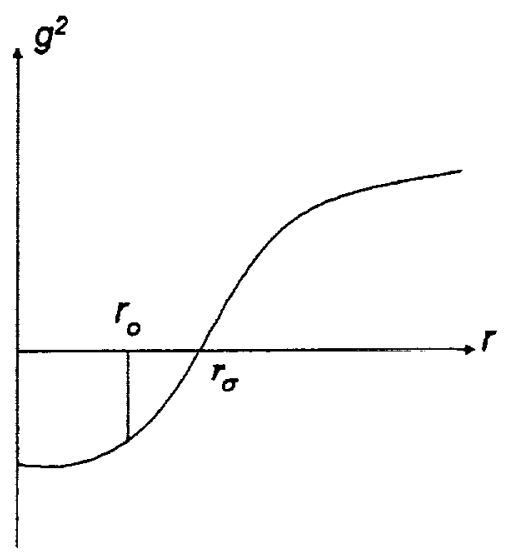

Fig. 3 One turming point problem.

Thus $f^{2}$ is positive when $r<r_{\sigma}$ (Fig. 3) and from (7d) it is seen that the last exponential factor results in noise attenuation which becomes stronger the deeper the source is embedded in the negative region of $g^{2}(r, \theta)$ and the higher its frequency. The case with two turning points is also discussed in the same references. In general, the shielding of noise depends on the proximity of the source with respect to the tuming point $r_{\sigma}$ as well as the number of turning points.

\subsection{Noise Sources}

The prediction of jet noise due to small-scale turbulence may be carried out according to Eq. (4) once the spectral density of a two-point correlation associated with various source terms of Lilley's equation are known. Any realistic computation of Eq. (4) requires some sort of acceptable modeling to facilitate a closed-form integration with respect to the separation vector $\bar{\xi}$. If the time history of source fluctuations is not known, additional modeling related to the temporal factor of a two-point correlation becomes necessary in order to evaluate the source spectral density. In this section, we discuss the two relatively more significant sources of Lilley's equation, namely self- and shear-noise terms as given in Eq. (2). 


\subsubsection{Self Noise}

To assess the far-field mean-square pressure due to the self-noise term only, we write (2) in a moving coordinate

$$
L\left(p_{s e l f} ; V, x_{1}^{\prime}\right)=D\left(\rho_{o} \frac{\partial^{2}\left(u_{i} u_{j}\right)}{\partial x_{i}^{\prime} \partial x_{j}^{\prime}}\right)
$$

where density $\rho_{o}$ has moved to the right of operator $D=\partial / \partial t+V \partial / \partial x^{\prime}$ assuming that flow is locally parallel and that density fluctuations are small so $\rho_{o}$ is the mean density at the source. We now write Lilley's Green's function $S$ for a source of type $D\left\{e^{-i \Omega} \delta\left(\vec{x}^{\prime}-\vec{x}_{o}^{\prime}\right)\right\}$

$$
L\left(S e^{-i \Omega} ; V, x_{1}^{\prime}\right)=D\left\{e^{-i \Omega t} \delta\left(x^{\prime}\right) \delta\left(\varphi-\varphi_{o}\right) \delta\left(r-r_{o}\right) / r\right\}
$$

It is easily shown that $S$ is related to $G$

$$
S\left(\vec{x}^{\prime}, \bar{y}, \Omega\right)=-i \Omega \frac{1-M_{o} \cos \theta}{1-M_{c} \cos \theta} G\left(\vec{x}^{\prime}, \bar{y}, \Omega\right) .
$$

The acoustic pressure due to the above source and Green's function becomes

$$
p_{\text {self }}(\bar{x}, t)=\iint_{i_{1}} S\left(\bar{x}^{\prime}, t ; \bar{y}, t_{1}\right) \rho_{o} \frac{\partial^{2}\left(u_{i} u_{j}\right)}{\partial y_{i} \partial y_{j}} d t_{1} \not \bar{y},
$$

where $S\left(\vec{x}^{\prime}, t ; \vec{y}, t_{1}\right)$ is inverse Fourier transform of $S\left(\vec{x}^{\prime}, \vec{y}, \Omega\right) e^{-i \Omega s}$. Integrating by parts with suitable restrictions on $S$ and its derivatives at large values of $y_{i}$ one finds

$$
p_{s e l f}(\vec{x}, t)=\iint_{i_{1}} u_{i} u_{j} \frac{\partial^{2}(\hat{S})}{\partial y_{i} \partial y_{j}} d t_{1} d \bar{y}, \quad \hat{S} \equiv \rho_{o} S .
$$

The compactness conditions requires $\Omega l / a_{\infty}$ to be small ( $l$ is a typical eddy length scale). Since frequency $\Omega$ is of the order of $v / l$ ( $v$ is the R.M.S. of the velocity fluctuations) then the compactness condition should be true if $v / a_{\infty}$ is small. Consequently, in fine-grain turbulence, the eddy length scale is expected to be much shorter than the wavelength of the acoustic disturbances $(l<<\lambda)$. If this is true, then mean square acoustic disturbances in the far field may be expressed as a superposition of disturbances from independently correlated volume elements in a jet

$$
\overline{p_{s e l f}^{2}}(\bar{x}, \Omega)=\int_{\bar{y}} \hat{S}_{, j}(\vec{x}, \vec{y}, \Omega) \hat{S}^{*},{ }_{k l} I_{i j k l}(\vec{y}, \Omega) d \vec{y},
$$

where

$$
I_{i j k l}(\vec{y}, \Omega) \equiv \int_{\xi}^{+\infty} \int_{\left(u_{i} u_{j}\right)\left(u_{k}^{\prime} u_{i}^{\prime}\right) e^{i \Omega \tau}} d \tau d \xi
$$

In (13b), the integrand of the source term $I_{i j k d}$ is the familiar fourth-order space-time correlation. The velocity variables with and without prime are evaluated at two points $\bar{y}_{1}$ and $\bar{y}_{2}$ separated by vector $\bar{\xi}$ and time-delay $\tau$. The factor multiplying the source intensity in (13a) defines a directivity factor that accounts for effects such as source convection and mean-flow refraction.

The volume integration in (13a) needs to include the most energetic parts of the jet. When the flow is axisymmetric, the directivity factor may be averaged azimuthally with respect to source and observer circumferential angles to obtain a ringsource directivity factor $a_{i j l l}$. Subsequently, jet volume integration will be limited to radial and axial coordinates

$$
\begin{aligned}
& a_{i j k l} \equiv \frac{1}{4 \pi^{2}} \int_{-\pi-\pi}^{+\pi+\pi} \int_{, j j} \hat{S}_{, j, k l} \hat{S}_{, k}^{*} d \varphi d \varphi_{o}, \\
& \overline{p_{s e l f}^{2}}(\vec{x}, \Omega)=\iint_{y_{1}} a_{i j k l} I_{i j k l}(\vec{y}, \Omega) d r d y_{1}
\end{aligned}
$$

Appendix A gives approximate expressions for $\hat{S}_{, i j}$ upon neglecting $\rho_{, r_{r_{o}} r_{o}}$ and $M_{, r_{o} r_{o}}$. Directivity factors $a_{i j k l}$ are now calculated by placing appropriate variables $\hat{S}_{, i j}$ into Eq. (14a). Of the 81 components, it tums out that some are equal and some are identically zero. When the shielding function $g^{2}(r)$ is positive for values of radial coordinate $r$ from source point $r_{o}$ to the observer point, the solution is referred to as unshielded and the corresponding directivity factors relevant to our calculations become

$$
\begin{aligned}
& a_{1111}=k^{4}|\hat{S}|^{2}\left(\frac{\cos \theta}{1-M_{c} \cos \theta}\right)^{4}, \\
& a_{1122}=\frac{1}{2} k^{2}|\hat{S}|^{2}\left(\frac{\cos \theta}{1-M_{c} \cos \theta}\right)^{2}\left(a_{1}-a_{2}+a_{3}\right), \\
& a_{1212}=\frac{1}{2} k^{2}|\hat{S}|^{2}\left(\frac{\cos \theta}{1-M_{c} \cos \theta}\right)^{2}\left[a_{1}+r_{o}^{2} a_{3}^{2}\right], \\
& a_{2222}=\frac{1}{4}|\hat{S}|^{2}\left[\frac{3}{2}\left(a_{1}^{2}+a_{2}^{2}+a_{3}^{2}\right)-2 a_{1} a_{2}-a_{2} a_{3}+2 a_{1} a_{3}+\right], \\
& a_{2233}^{2}+b_{2}^{2}-b_{1} b_{2} \\
& a_{2323}=\frac{1}{4}|\hat{S}|^{2}\left[\frac{1}{2}\left(a_{1}^{2}+a_{2}^{2}+a_{3}^{2}\right)-2 a_{1} a_{2}+2 a_{1} a_{3}-3 a_{2} a_{3}-\right], \\
& b_{1}^{2}+b_{1} b_{2}
\end{aligned}
$$


Factors $a_{i}$ and $b_{i}$ have a dimension of $L^{-2}$ and depend on the mean flow properties as

$$
\begin{aligned}
& a_{1}=k^{2} g_{o}^{2}, \quad a_{2}=\mathrm{N}, \quad a_{3}=\frac{1}{r_{o}}\left(\mathrm{~T}_{1}-\frac{g_{r_{o}}}{2 g_{o}}\right), \\
& b_{1}=-2 k g_{o} \mathrm{~T}_{1}, \quad b_{2}=\frac{1}{2} k g_{, r_{o}} .
\end{aligned}
$$

Variables $a_{2}, a_{3}, b_{1}$ and $b_{2}$ become identically zero when gradient terms $\rho_{, r_{0}}$ and $M_{, r_{0}}$ are neglected (see Appendix A), and hence the directivity factors (15a) reduce to those given in [11].

When $g^{2}(r)$ becomes negative at the source, a shielding zone exists. The amount of shielding depends on the proximity of source with respect to the turning point $r_{\sigma}$ as well as the number of turning points. This dependence is accounted for by multiplying directivity factors (15a) by a shielding coefficient $\beta_{i j}$. For example when there is only one tuming point $r_{\sigma 1}$, the shielding coefficient becomes

$$
\beta_{12}=e^{-2 k \int_{r_{0}}^{r_{1} 1} \sqrt{\left|g^{2}(r)\right|} d r}
$$

Eq. (15a) is now written for a unit volume ring within a turbulent jet

$$
\begin{aligned}
\overline{p_{\text {self }}^{2}}(\vec{x}, \Omega ; \vec{y})= & a_{1111} I_{1111}+4 a_{1122} I_{1122}+8 a_{1212} I_{1212}+ \\
& 2 a_{2222} I_{2222}+2 a_{2233} I_{2233}+4 a_{2323} I_{2323}
\end{aligned}
$$

\subsubsection{Source Correlations Tensors}

Self noise is evahuated by integrating (16) for the most energetic parts of a turbulent jet. Source correlation tensors $I_{i j k t}(\bar{y}, \Omega)$ are usually calculated using a physics-based modeling approach. As was done in Ref. [11], we assume that turbulence is axisymmetric about the direction of the mean flow. Various source correlation tensors are simplified and written in terms of the axial component $I_{1111}$ (see Appendix B)

$$
I_{1111}(\bar{y}, \Omega)=\left(\overline{u_{1}^{2}}\right)^{2} \frac{L_{1}^{3} \Delta^{2}}{2 \sqrt{2}} \int_{-\infty}^{+\infty} g^{2}(\tau) e^{i \Omega \tau} d \tau .
$$

Here $g(\tau)$ is the temporal part of a two-point correlation $\overline{u_{i} u_{j}^{\prime}}$, $L_{1}$ and $L_{2}$ are axial and transverse length scales of an axisymmetric turbulence and $\Delta=L_{2} / L_{1}$. Parameter $\beta=\left(1-\overline{u_{2}^{2}} / \overline{u_{1}^{2}}\right)$ is assumed known $a$ priori, and the axial component of turbulence intensity is expressed as $\overline{u_{1}^{2}}=\frac{2}{3} \kappa /\left(1-\frac{2}{3} \beta\right)$ where $\kappa$ is the turbulence kinetic energy.
Further, using the usual assumptions we relate the axial length scale $L_{1}$ to the turbulence kinetic energy and its dissipation rate $\varepsilon$ as $L_{1} \sim\left(\overline{u_{1}^{2}}\right)^{3 / 2} / \varepsilon$. In addition, the source characteristic frequency $\Omega_{0}$ (which is the inverse of characteristic time-delay $\tau_{0}$ ) is written as $\Omega_{0} \sim \varepsilon / K$. Time-delay function of a twopoint correlation was selected as $g(\tau)=\exp \left(-\sqrt{(\sigma / 2)^{2}+\left(\tau / \tau_{o}\right)^{2}}\right)$ with $\sigma=0.8$. Making the necessary substitutions, we find from (17)

$$
I_{1111}(\vec{y}, \Omega) \sim \kappa^{7 / 2} \tau_{o}^{4} \frac{K_{1}\left(\sigma \sqrt{1+\left(\Omega \tau_{o} / 2\right)^{2}}\right)}{\sqrt{1+\left(\Omega \tau_{o} / 2\right)^{2}}} \Delta^{2}\left(1-\frac{2}{3} \beta\right)^{-13 / 2},
$$

where $K_{1}$ is a modified Bessel function of the second kind and of order one. A Doppler factor relates the source frequency $\Omega$ to the observer frequency $\omega$ as

$$
\Omega=\omega \sqrt{\left(1-M_{c} \cos \theta\right)^{2}+\left(\alpha_{c} \kappa^{0.5} / a_{\infty}\right)^{2}},
$$

where $\alpha_{c}$ is an empirical constant selected as 0.50 .

\subsubsection{Shear Noise}

The sound field due to shear-noise terms of Lilley's Eq. (see Eq. 2) is given using the Green's function of Eq. (7)

$$
p_{\text {shear }}(\vec{x}, t)=-2 \iint_{i_{1}} G\left(\vec{x}^{\prime}, t ; \vec{y}, t_{1}\right) \rho \frac{d U}{d r} \frac{\partial^{2}\left(u_{2} u_{j}\right)}{\partial y_{1} \partial y_{j}} d t_{1} d \vec{y}
$$

It is implied that subscript 2 on the source term $u_{2} u_{j}$ refers to a transverse direction, which when expanded is replaced with $u_{2} u_{j}+u_{3} u_{j}$. Integration by parts results in

$$
p_{\text {sheor }}\left(\vec{x}^{\prime}, t\right)=-2 \iint_{i_{1}} u_{\bar{y}} u_{j} \frac{\partial}{\partial y_{j}}\left(\rho \frac{d U}{d r} \frac{\partial G}{\partial y_{1}}\right) d t_{1} d \vec{y} .
$$

In writing (20), we have used the parallel flow assumption $\partial \rho / \partial y_{1}=0$, and $\partial U / \partial y_{1}=0$. Let $\hat{G}$ denote the quantity inside the bracket in the last equation. Upon recognizing that $\partial G / \partial y_{1}=i k G \cos \theta /\left(1-M_{c} \cos \theta\right)$, we write

$$
\begin{aligned}
& \hat{G} \equiv \rho_{o} \frac{d U}{d r} \frac{\partial G}{\partial y_{1}}, \\
& b_{i j} \equiv \frac{2^{2}}{4 \pi^{2}} \int_{-\pi-\pi}^{+\pi+\pi} \int_{,_{i}} \hat{G}_{, j}^{*} d \varphi d \varphi_{o}, \\
& \overline{p_{\text {shear }}^{2}}(\bar{x}, \Omega)=\iint_{y_{1}, r} b_{i j} I_{2 i 2 j}(\bar{y}, \Omega) d r d y_{1} .
\end{aligned}
$$


Upon neglecting the second derivative $U_{, r}$, expressions for $\hat{G}_{, i}$ become

$$
\begin{gathered}
\left(\begin{array}{l}
\hat{G}_{.1} \\
\hat{G}_{, 2} \\
\hat{G}_{, 3}
\end{array}\right)=i k\left(\begin{array}{c}
\frac{\cos \theta}{1-M_{c} \cos \theta} \\
-g_{o} \cos \varphi \\
-g_{o} \sin \varphi
\end{array}\right) \hat{G}-\left(\begin{array}{c}
0 \\
\mathrm{~T}_{3} \cos \varphi_{o} \\
\mathrm{~T}_{3} \sin \varphi_{o}
\end{array}\right) \hat{G}, \\
\mathrm{~T}_{3}=\frac{\rho_{, r_{o}}}{2 \rho_{o}}+2 \frac{M_{, r_{o}} \cos \theta}{1-M_{o} \cos \theta}-\frac{g_{, r_{o}}}{2 g_{o}} .
\end{gathered}
$$

and

Using (22a) in (21), shear noise due to a unit volume ring source becomes

$$
\overline{p_{\text {shear }}^{2}}(\vec{x}, \Omega ; \vec{y})=4\left(b_{11} I_{2121}+b_{12} I_{2222}+b_{13} I_{2323}\right),
$$

and the corresponding unshielded directivity factors are

$$
\begin{aligned}
& b_{11}=k^{4}\left(\frac{\cos \theta}{1-M_{c} \cos \theta}\right)^{4}\left(\frac{d U}{d r}\right)^{2} \rho_{o}^{2}|G|^{2}, \\
& b_{12}=\frac{1}{2} k^{2}\left(\mathrm{~T}_{3}^{2}+k^{2} g_{o}^{2}\right)\left(\frac{\cos \theta}{1-M_{c} \cos \theta}\right)^{2}\left(\frac{d U}{d r}\right)^{2} \rho_{o}^{2}|G|^{2}, \\
& b_{13}=b_{12} .
\end{aligned}
$$

For the shielded case, (i.e. when $g^{2}\left(r_{o}\right)<0$, and $r_{o}<r_{\sigma}$ ), shielding factor $\beta_{i j}$ as discussed earlier should multiply the above directivity factors.

When gradients $\rho_{, r_{o}}$ and $M_{r_{0}}$ are neglected, it is readily seen that the directivity factors of shear noise also recuce to those given in Ref. [11].

\section{Numerical Results}

A single stream Round-Convergent nozzle (RC) with an exit diameter of $D=12.13 \mathrm{~cm}$. is selected for numerical simulation (Fig. 3). RANS calculations are performed with the NPARC Navier-Stokes solver [17] and Chien's k- $\varepsilon$ turbulence model [18]. Inflow conditions are specified at inlet ( $4.33 \mathrm{D}$ from nozzle exit), and downstream conditions are provided at $20 D$ from exit plane. In a spanwise direction, the grid extends $8.11 D$ from the centerline, and a free-type boundary condition is specified. Our primary objective here is to examine the effect of temperature, or equivalently the mean density gradient on radiated jet noise. A set of run points for the CFD predictions are carefully selected with the intention of changing the jet temperature while maintaining its velocity.

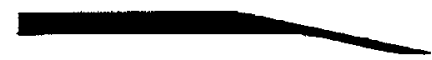

Fig. 3 RC nozzle geometry.

A total of four run points are shown here. Table la shows that by lowering the nozzle pressure ratio (NPR) from 1.4 to 127 , and at the same time increasing upstream total temperature $T_{o} / T_{\infty}$ from 1.55 to 2.27 , the jet exit velocity may be kept constant at $U_{j} / a_{\infty}=0.83$ while exit temperature $T_{j} / T_{\infty}$ is increased from 1.42 to 2.13. Similar nun points are shown in Table $\mathrm{lb}$ for a low subsonic jet velocity of $U_{j} / a_{\infty}=0.52$

Table 1a Inflow conditions for exit velocity of $U_{j} / a_{\infty}=0.83$

\begin{tabular}{|c|c|c|c|c|}
\hline & \multicolumn{2}{|c|}{$\begin{array}{c}\text { Inflow Conditions } \\
\text { (input) }\end{array}$} & \multicolumn{2}{c|}{$\begin{array}{c}\text { Exit Conditions } \\
\text { (calculated) }\end{array}$} \\
\hline Case & NPR & $T_{o} / T_{\infty}$ & $U_{j} / a_{\infty}$ & $T_{j} / T_{\infty}$ \\
\hline 1 & 1.40 & 1.55 & 0.83 & 1.42 \\
\hline 2 & 127 & 2.27 & 0.83 & 2.13 \\
\hline
\end{tabular}

Table $1 \mathrm{~b}$ Inflow conditions for exit velocity of $U_{J} / a_{\infty}=0.52$

\begin{tabular}{|c|c|c|c|c|}
\hline & \multicolumn{2}{|c|}{$\begin{array}{c}\text { Inflow Conditions } \\
\text { (input) }\end{array}$} & \multicolumn{2}{c|}{$\begin{array}{c}\text { Exit Conditions } \\
\text { (calculated) }\end{array}$} \\
\hline Case & NPR & $T_{0} / T_{\infty}$ & $U_{j} / a_{\infty}$ & $T_{j} / T_{\infty}$ \\
\hline 3 & 1.14 & 1.55 & 0.52 & 1.50 \\
\hline 4 & 1.095 & 2.27 & 0.52 & 2.21 \\
\hline
\end{tabular}

Predicted radial profiles for mean velocity, temperature, and density are shown (Fig. 4) at the intermediate jet exit velocity of $U_{j} / a_{\infty}=0.83$ and at four streamwise locations along the jet axis; hence the effect of heating on jet noise is examined by eliminating velocity dependence from calculations.

Noise predictions were performed at subsonic conditions of Tables la and $1 \mathrm{~b}$, and compared with experimental data [13]. Measurements of Tanna [13] were conducted for a convergent nozzle with exit diameter of $5.08 \mathrm{~cm}$ and on an arc at 72 diameters from exit. Although the details of the geometry and run points for our numerical simulation are not exactly the same as the experiment, for all practical purposes a similar parametric study is conducted and a qualitative comparison is justifiable. For example, measurements indicate that at high frequency, mixing noise decreases with heating the jet throughout the jet velocity (see Fig. 5). Low frequency noise increases with heating at subsonic acoustic Mach numbers $U_{j} / a_{\infty}$ (Fig. 5), while it decreases with heating at supersonic Mach numbers (not shown here). Measured directivity (Fig. 6) indicates an overall rectuction 
in noise with heating at high and intermediate velocity and an increase in noise at low subsonic Mach number of $U_{j} / a_{\infty}=0.50$.

Figures 7 and 8 show the effect of heating on the predicted spectra at intermediate and low jet velocities of $U_{j} / a_{\infty}=0.83$ and 0.52 . The spectra are computed at $R D=125$. Clearly the temperature gap between the jets in our simulation (Table 1) is not as wide as in the experiment, yet an increase in low frequency noise and a reduction in high frequency noise is still evident. The integrated spectra (Fig. 9) shows a reduction in SPL with increasing temperature at all angles. At low Mach number of Fig.10, the directivity factor does not exactly follow the trend suggested by data. Comparing figures 7 and 8 with data of Fig. 5, it is observed that the increase in low frequency noise in our simulation in not as large as suggested by data. However, the density gradient terms provide improvements at the low end of the spectra and suggest a trend more compatible with measurement. Figures 11 and 12 demonstrate the effect of heating on noise directivity at specified Strouhal numbers $\left(S-J D / J_{j}\right)$. They show that at low frequency, heating results in a relatively small increase in noise intensity at mid angles. At high frequency, addition of heat reduces the noise and increasingly more so at a higher jet velocity. Fig. 13 examines the contributions from multi-pole components of self and shear noise. Noise components Q, D and $\mathrm{M}$ denote the quadrupole, dipole and monopole predictions, which are proportional to $k^{4}, k^{2}$, and $k^{0}$ respectively. When density gradient terms are neglected, only the quadrupole contributions are present. Outside that, the new sources appear primarily at the low end of the spectra.

Further increase in jet speed in the present numerical simulation requires a shock-free convergent-divergent nozzles (CD). An increase in the jet temperature at a given supersonic speed requires a new design Mach number and hence a new nozzle geometry. Measurements [13] indicate that noise level should drop with heat at the entire range of the frequency spectrum.

\section{Conclusions}

This study was motivated by a desire to examine the effect of heating on jet noise spectra. In addition to the mean flow refraction effects that directly influence the directivity of jet noise, new sources related to the radial gradients of mean density and sound speed were introduced. A physics-based modeling approach was developed to approximate self- and shear-noise sources of Lilley's equations. Turbulent velocity fluctuations were assumed axisymmetric about the direction of the mean flow. The present model enhanced the prediction capabilities of jet mixing noise due to small-scale turbulence. It was suggested that outside the usual quadrupoles, contributions from newly calculated sources were mainly due to dipole sources of self noise and at the low end of the frequency spectrum.

\section{Appendix A}

Various derivatives of $\hat{S}$ are needed with respect to source coordinates $y_{i}$. We use the equation of state for an ideal gas and express the sound speed as $a_{0}^{2}=P \gamma / \rho_{o}$, where $P$ is a constant static pressure; hence $\hat{S}$ is proportional to $\sqrt{\rho_{0}}$. In addition, the radial gradients of the Mach number and sound speed appearing in the Green's function (see Eq. 7) are accounted for up to the first derivative. A transformation is made to a polarcylindrical coordinate system

$$
\left(\begin{array}{l}
\partial / \partial y_{2} \\
\partial / \partial y_{3}
\end{array}\right)=\left(\begin{array}{cc}
\cos \varphi_{o} & -\sin \varphi_{o} \\
\sin \varphi_{o} & \cos \varphi_{o}
\end{array}\right)\left(\begin{array}{c}
\partial / \partial r_{o} \\
\frac{\partial}{r_{0} \partial \varphi_{o}}
\end{array}\right) .
$$

The first derivatives become

$$
\left(\begin{array}{l}
\hat{S}_{, 1} \\
\hat{S}_{, 2} \\
\hat{S}_{, 3}
\end{array}\right)=i k\left(\begin{array}{c}
\frac{\cos \theta}{1-M_{c} \cos \theta} \\
-g_{0} \cos \varphi \\
-g_{o} \sin \varphi
\end{array}\right) \hat{S}+\left(\mathrm{T}_{1}-\frac{1}{2} \frac{g_{, r_{0}}}{g_{0}}\right)\left(\begin{array}{c}
0 \\
\cos \varphi_{o} \\
\sin \varphi_{0}
\end{array}\right) \hat{S},
$$

where

$$
\begin{aligned}
& g_{, r_{o}}=-\frac{\left(a_{\infty} / a_{o}\right)^{2}}{g_{o}}\left(\frac{1-M_{o} \cos \theta}{1-M_{c} \cos \theta}\right)^{2} \mathrm{~T}_{2}, \\
& \mathrm{~T}_{n}=\frac{\rho_{, r_{o}}}{2 \rho_{o}}-(-1)^{n} \frac{M_{, r_{o}} \cos \theta}{1-M_{o} \cos \theta}, \quad n=1,2
\end{aligned}
$$

Upon neglecting $\rho_{, r_{o} r_{0}}$ and $M_{, r_{o} r_{o}}$, the second derivatives of $\hat{S}$ become

$$
\left(\begin{array}{l}
\hat{S}_{.11} \\
\hat{S}_{.22} \\
\hat{S}_{, 33} \\
\hat{S}_{, 23}
\end{array}\right)=-k^{2}\left(\begin{array}{c}
\frac{\cos ^{2} \theta}{\left(1-M_{c} \cos \theta\right)^{2}} \\
g_{o}^{2} \cos ^{2} \varphi \\
g_{o}^{2} \sin ^{2} \varphi \\
\frac{g_{o}^{2}}{2} \sin 2 \varphi
\end{array}\right) \hat{S}+\mathrm{N}\left(\begin{array}{c}
0 \\
\cos ^{2} \varphi_{o} \\
\sin ^{2} \varphi_{o} \\
\frac{1}{2} \sin 2 \varphi_{o}
\end{array}\right) \hat{S}
$$

$+\frac{1}{r_{o}}\left(\mathrm{~T}_{1}-\frac{1}{2} \frac{\left.g_{,_{o}}\right)}{g_{o}}\left(\begin{array}{c}0 \\ \sin ^{2} \varphi_{o} \\ \cos ^{2} \varphi_{o} \\ -\frac{1}{2} \sin 2 \varphi_{o}\end{array}\right) \hat{S}-2 i k g_{o} \mathrm{~T}_{1}\left(\begin{array}{c}0 \\ \cos \varphi \cos \varphi_{o} \\ \sin \varphi \sin \varphi_{o} \\ \frac{1}{2} \sin \left(\varphi+\varphi_{o}\right)\end{array}\right) \hat{S}\right.$

$+\frac{i}{2} k g_{, r_{o}} \sin \left(\varphi+\varphi_{o}\right)\left(\begin{array}{c}0 \\ -\sin 2 \varphi_{o} \\ \sin 2 \varphi_{o} \\ \cos 2 \varphi_{o}\end{array}\right) \hat{S}$, 
and $\mathrm{N}$ is given as

$$
\begin{aligned}
\mathrm{N}= & \mathrm{T}_{1}^{2}+\frac{\partial \mathrm{T}_{1}}{\partial r_{0}}+\left(\frac{a_{r_{o}}}{a_{0}}+\frac{5}{4} \frac{g_{, r_{o}}}{g_{o}}+\frac{M_{, r_{o}} \cos \theta}{1-M_{o} \cos \theta}\right. \\
& \left.-\frac{1}{2 \mathrm{~T}_{2}} \frac{\partial \mathrm{T}_{2}}{\partial r_{0}}-\mathrm{T}_{1}\right) \frac{g_{, r_{0}}}{g_{0}},
\end{aligned}
$$

Once the ratio $a_{, r_{0}} / a_{o}$ is replaced with $-\rho_{, r_{0}} /\left(2 \rho_{o}\right)$ and radial gradients of variables $T_{1}$ and $T_{2}$ become

$$
\frac{\partial \mathrm{T}_{n}}{\partial r_{o}}=-\frac{1}{2}\left(\frac{\rho_{, r_{o}}}{\rho_{o}}\right)^{2}-(-1)^{n}\left(\frac{M_{, r_{o}} \cos \theta}{1-M_{o} \cos \theta}\right)^{2}, \quad n=1,2
$$

\section{Appendix B}

Various source correlation tensor $I_{i j k d}$ are related to the axial tensor $I_{111}$

$$
\begin{aligned}
& I_{2222}=I_{3333}=C_{1} I_{1111,} \\
& I_{1122}=I_{1133}=C_{2} I_{1111,} \\
& I_{2233}=C_{3} I_{1111}, \\
& I_{1313}=I_{1212}=C_{4} I_{1111,} \\
& I_{2323}=C_{5} I_{1111} .
\end{aligned}
$$

For an axisymmetric turbulence coefficients $C_{i}$ are given as [11]

$$
\begin{aligned}
\Delta & =\frac{L_{2}}{L_{1}}, \quad \beta=1-\frac{\overline{u_{2}^{2}}}{u_{1}^{2}}, \\
C_{1} & =\frac{3}{2} \beta^{2}-\frac{\beta}{4}\left(6-\Delta^{2}+3 \Delta^{-2}\right)+\frac{1}{32}\left[9\left(\Delta+\Delta^{-1}\right)^{4}\right. \\
& \left.-48\left(\Delta+\Delta^{-1}\right)^{2}+80\right], \\
C_{3} & =\frac{1}{2} \beta^{2}+\frac{\beta}{4}\left(-2+\Delta^{2}-\Delta^{-2}\right)+\frac{1}{8}\left[\frac{3}{4}\left(\Delta+\Delta^{-1}\right)^{4}\right. \\
& \left.-4\left(\Delta+\Delta^{-1}\right)^{2}+7-2 \Delta^{2}\right], \\
C_{2} & =\frac{\Delta^{2}}{8}, \quad C_{4}=-\frac{\beta}{2}+\frac{1}{16}\left[2\left(\Delta+\Delta^{-1}\right)^{2}-\Delta^{2}\right], \\
C_{5} & =\frac{C_{1}-C_{3}}{2} .
\end{aligned}
$$

\section{Acknowledgement}

This Work was sponsored by NASA Glenn Research Center under the contract NAS3-98008.

\section{References}

'Lele, S. K., "Computational Aeroacoustics: A Review," AIAA 97-0018, January 1997.

${ }^{2}$ Goodrich, J. W., "A Comparison of Numerical Methods for Computational Aeroacoustics," AIAA 99-1943, May 1999.

${ }^{3}$ Goldstein, M. E., Aeroacoustics, McGraw Hill, 1976.

${ }^{4}$ Pilon, A. R. and Morris, P. J., "A Semi Analytical Method for Jet Noise Prediction," AIAA 99-0076, January 1999.

'Tam, C. K. W., Golebiowski, M., and Seiner, J. M., "On the Two Components of Turbulent Mixing Noise from Supersonic Jets," AIAA 96-1716, May 1996.

'Tam, C. K. W., and Auriault, L., "Jet Mixing Noise from Fine-Scale Turbulence," AIAA Journal, Vol. 37, No. 2; Feb. 1999, pp.145-153.

${ }^{7}$ Mitchell, B. E., Lele, S. K, and Moin, P., "Direct Computation of the Sound Generated by Subsonic and Supersonic Axisymmetric Jets," Technical ReportTF-66 Stanford University, 1995.

${ }^{8}$ Freund, J. B., Moin, P., and Lele, S. K, "Results of a Compressible Round Shear Layer Direct Simulation,"AIAA 97 $-0760,1997$.

'Mankbadi, R R., Hayder, M. E., and Povinelli, L.A.,"Structure of Supersonic Jet Flow and Its radiated Sound, AIAA Journal, Vol. 32, No. 5, May 1994, pp. 897-906.

${ }^{10}$ Morris, P. J., Wang, Q., Long, L. N., and Lockard, D. P., "Numerical Prediction of High Speed Jet Noise," AIAA 971598, May 1997.

${ }^{11}$ Khavaran, A., "Role of Anisotropy in Turbulent Mixing Noise," AIAA Joumal, Vol. 37, No.7, July 1999, pp. 832-841.

${ }^{12}$ Hoch, R. G., Duponchel, J. P., Cocking. B. J., and Bryce, W. D., "Studies of the Influence of Density on Jet Noise," Journal of Sound \& Vibration, Vol. 28, No. 4, 1973, pp. 649-668.

${ }^{13}$ Tanna, H. K. "An Experimental Study of Jet Noise, Part I: Turbulent Mixing Noise," Journal of Sound \& Vibration, Vol. 50, No. 3, 1977, pp. 405-428.

${ }^{14}$ Balsa, T. F., "The Far-Field of High Frequency Convected Singularities in Sheared Flows, with an Application to Jet Noise Prediction," Journal of Fluid Mechanics, Vol. 74, March 1976, pp. 193-208.

IS Mani, R, Gliebe, P. R, and Balsa, T. F., "High Velocity Jet Noise Source Location and Reduction, 'Federal Aviation Administration, Task 2, FAA-RD-76-II, Cincinnati, OH, May 1978.

${ }^{16}$ Balsa, T. F., "The Acoustic Field of Sources in Shear Flow with Application to Jet Noise: Convective Amplification," Journal of Fluid Mechanics, Vol. 79, part 1, 1977, pp. 33-47.

${ }^{17}$ Cooper, G. K, and Sirbaugh, J. R., "PARC Code: Theory and Usage," Amold Engineering Development Center Rept,AEDCTR-89-15, Tullahoma, TN, Dec. 1989.

${ }^{18}$ Chien, K. Y. "predictions of Channel and Boundary-Layer Flows with a Low-Reynolds-Number Turbulence Model," AIAA Journal, Vol 20, No. 1, 1982, pp. 33-38 

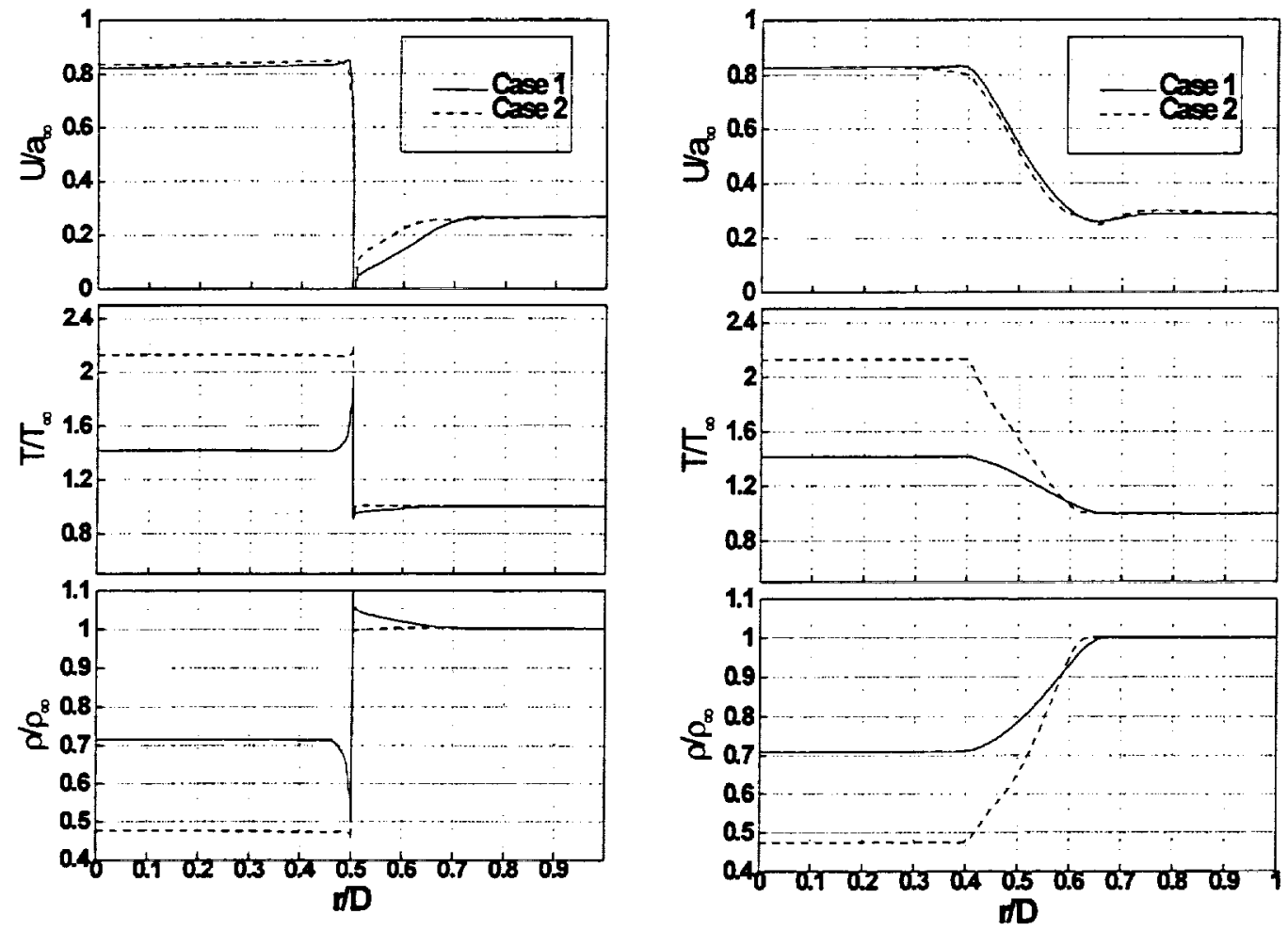

a) $X / D=0.0$.

b) $X / D=2.0$.
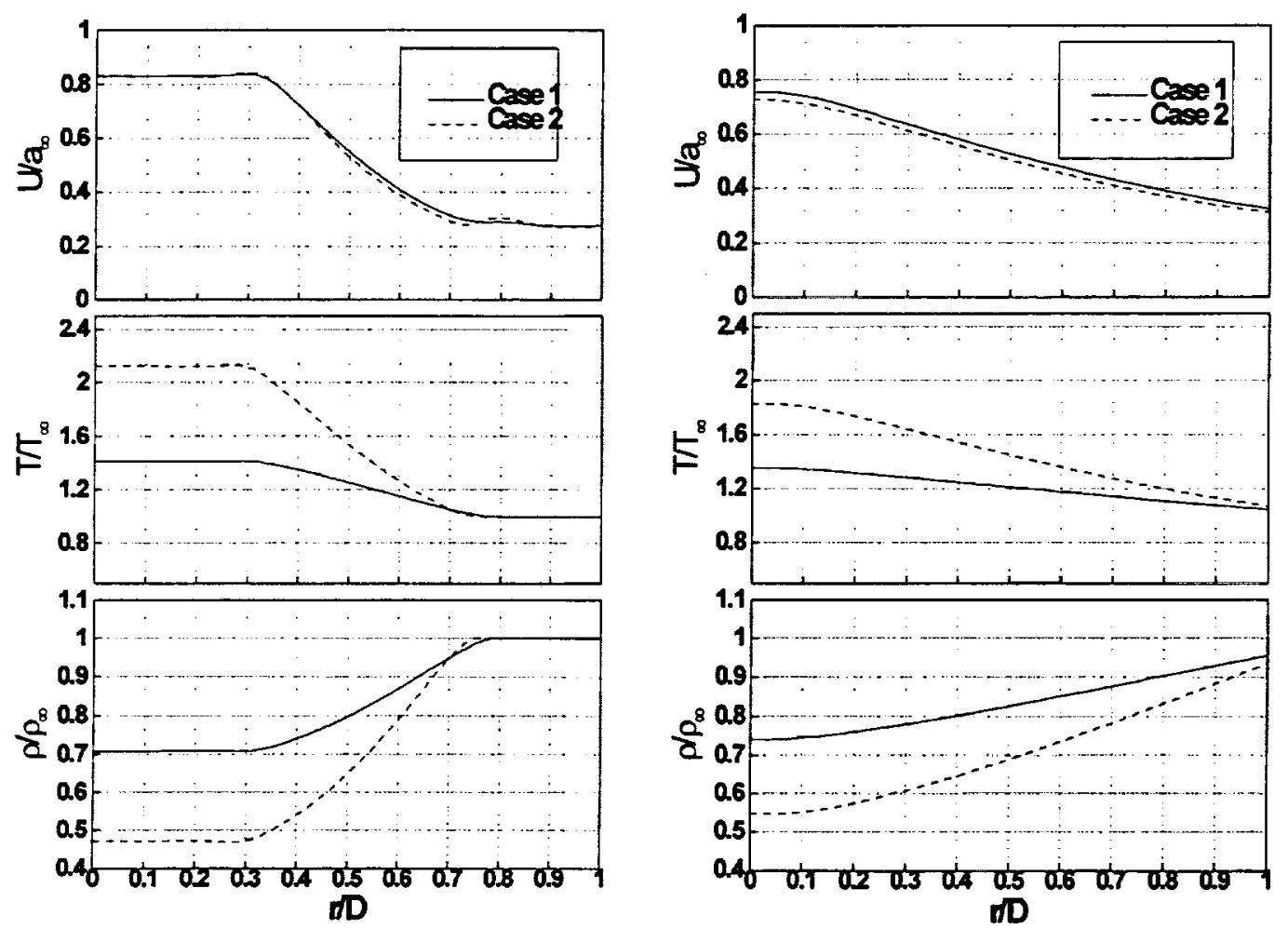

c) $X / D=4.17$.

d) $X / D=12.23$.

Fig. 4 Comparison of mean flow profiles for conditions of Table la and at indicated streamwise locations. 

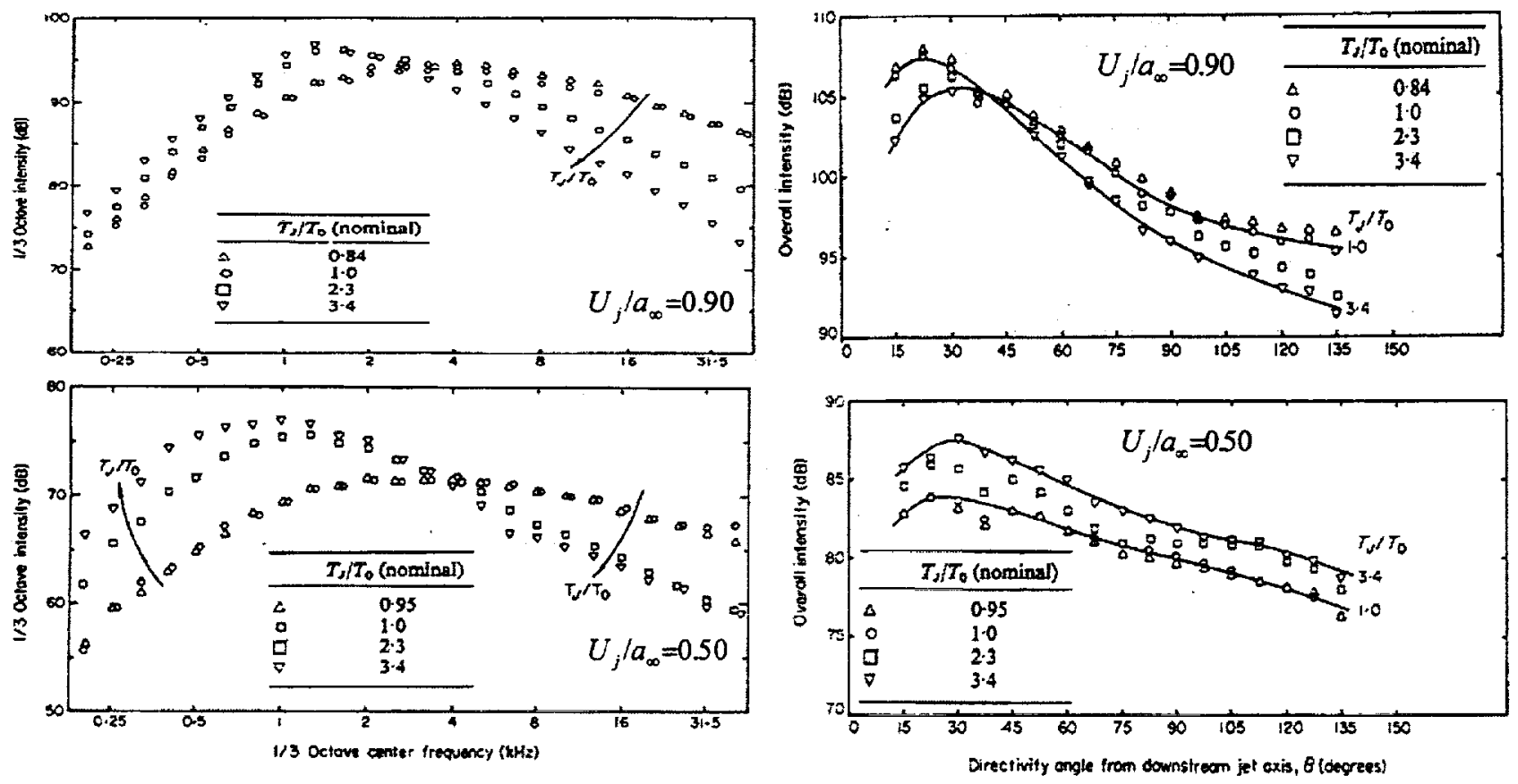

Fig. 5 Effect of temperature on noise spectra (measured data [13]) at 45 degrees from jet axis and at indicated jet exit velocities. 

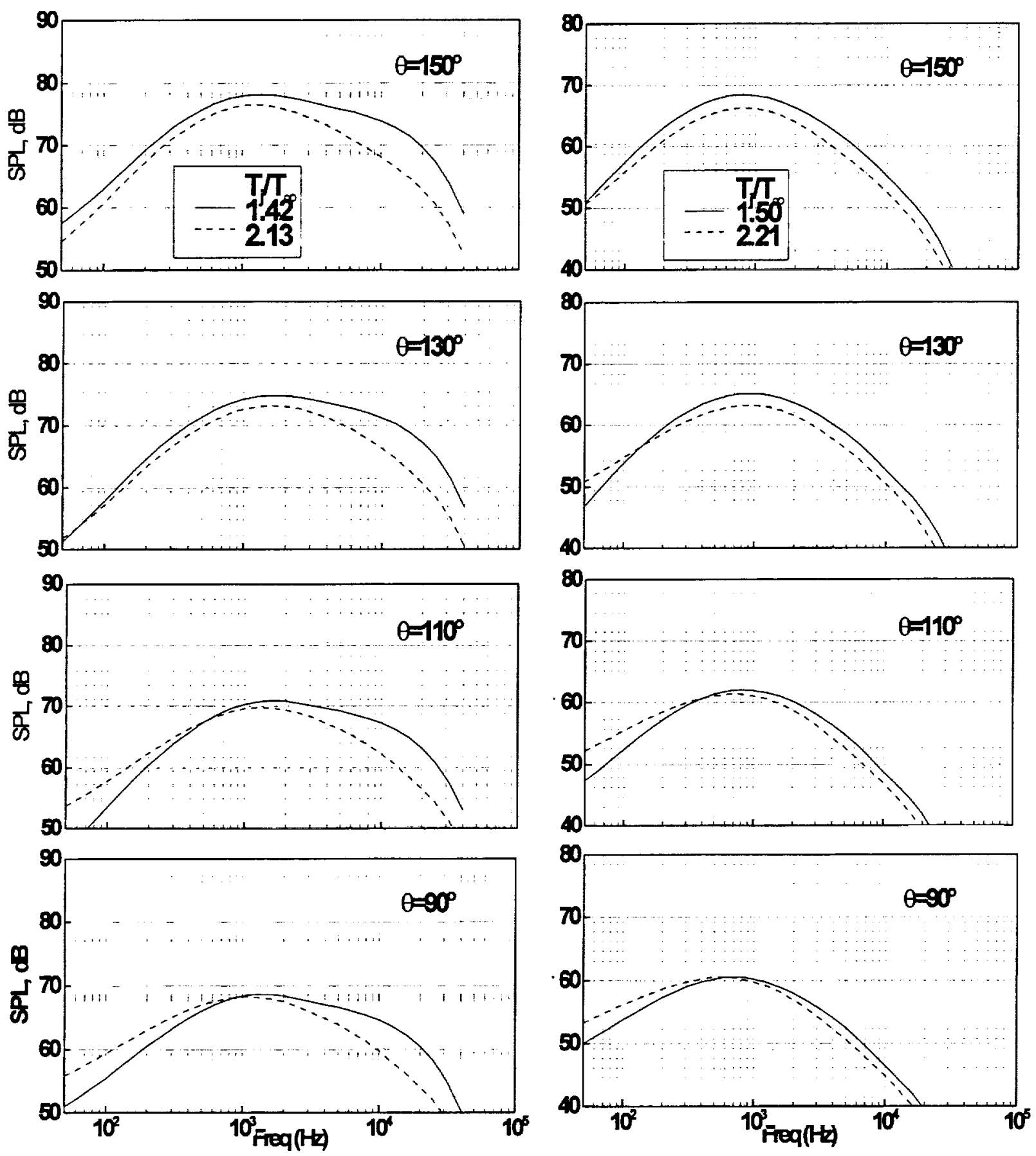

Fig. 7 Effect of temperature on noise spectra at $U_{j} / a_{\infty}=0.83, M_{\infty}=0.28$. Predictions on arc $R / D=125$ and with non-isotropy parameters $\Delta=0.87$, and $\overline{u_{2}^{2}} / \overline{u_{1}^{2}}=0.70$. Angle $\theta$ is from nozzle inlet.

Fig. 8 Effect of temperature on noise spectra at $U_{j} / a_{\infty}=0.52, M_{\infty}=0.07$. Non-isotropy parameters are as in Fig. 7. 


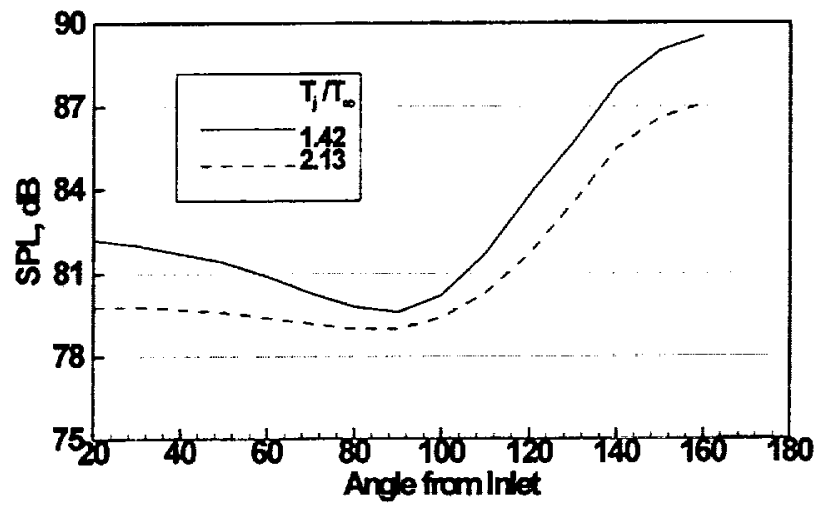

Fig. 9 Effect of temperature on noise directivity.

$\left(U_{j} / a_{\infty}=0.83, R D=125, \Delta=0.87, \overline{u_{2}^{2}} / \overline{u_{1}^{2}}=0.70\right)$
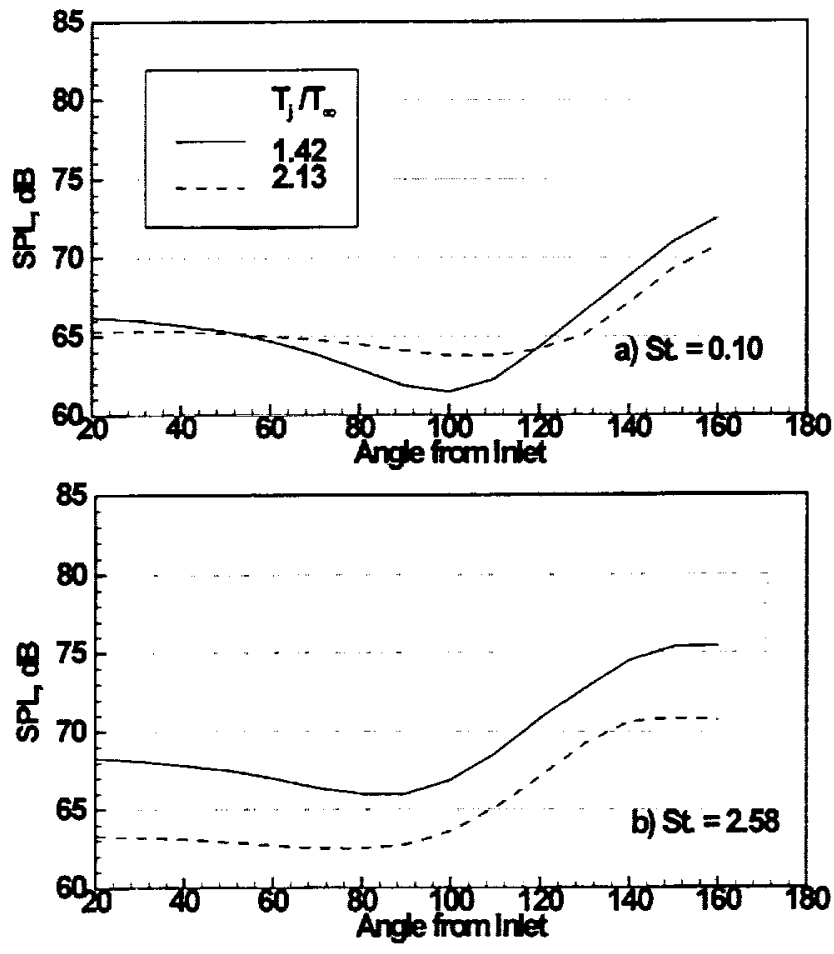

Fig. 11 Effect of temperature on noise directivity at indicated Strouhal numbers $S t=f D / U_{j}$ and for conditions of Figure 9.

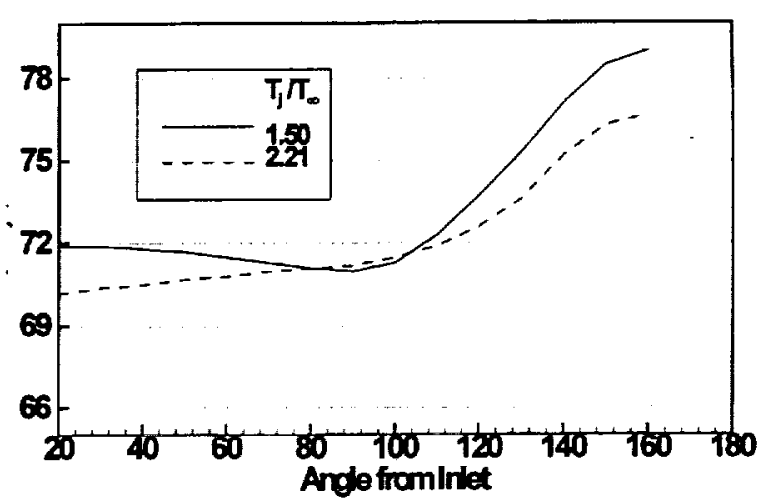

Fig. 10 Effect of temperature on noise directivity $\left(U_{j} / a_{\infty}=0.52, R D=125, \Delta=0.87, \overline{u_{2}^{2}} / \overline{u_{1}^{2}}=0.70\right)$
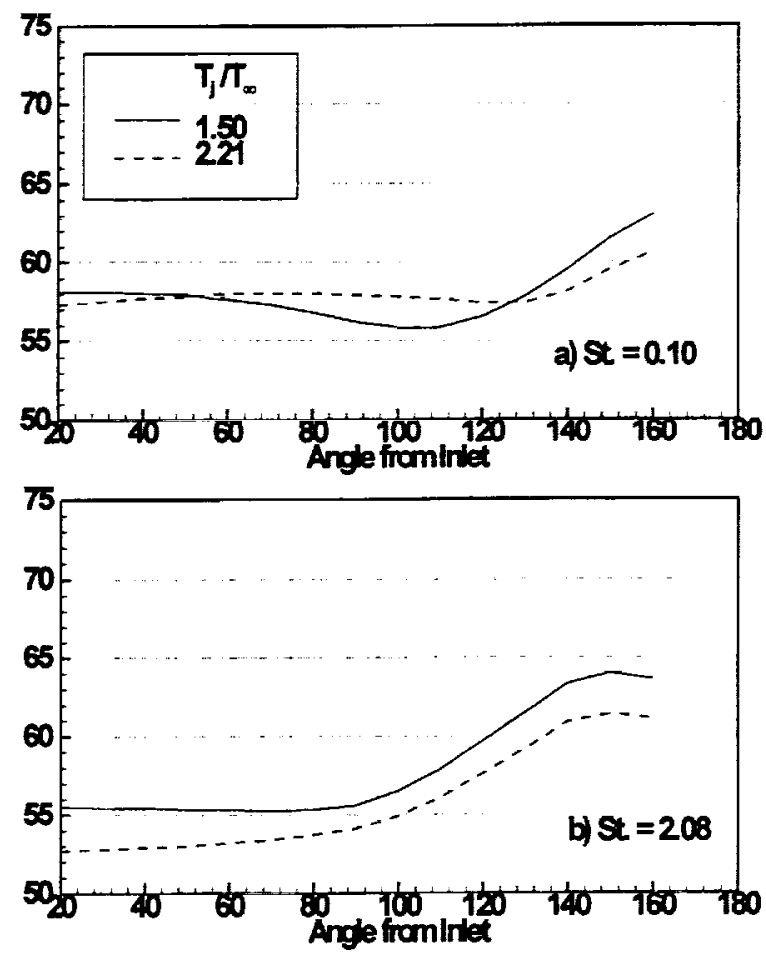

Fig. 12Effect of temperature on noise directivity at indicated Strouhal numbers and for conditions of Figure 10. 

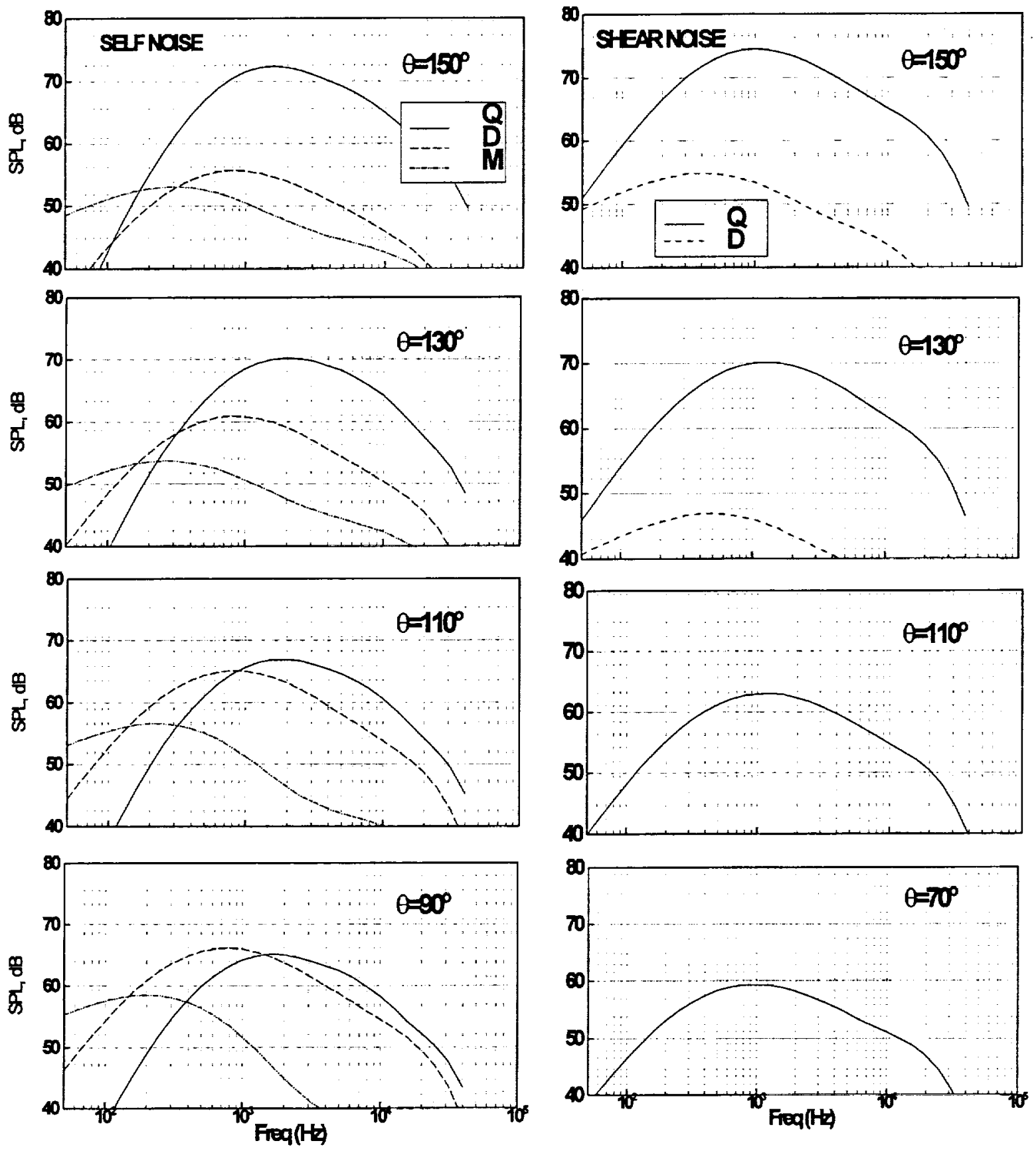

Fig. 13a. Predicted multi-pole components of self noise at operating conditions of Table. 1a case 2.

Fig. 13b. Predicted multi-pole components of shear noise at operating conditions of Table.1a case 2. 


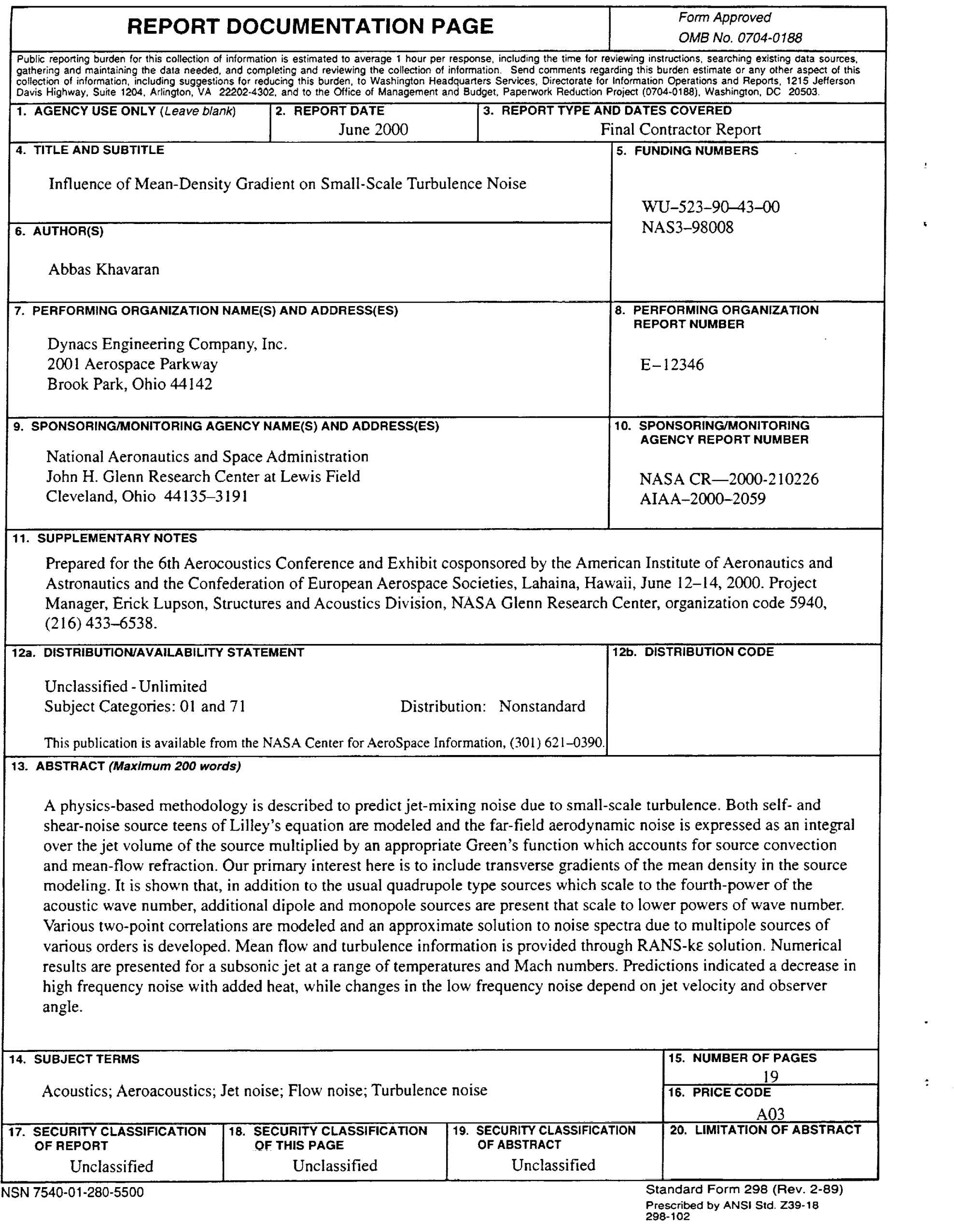

